\title{
Cooperative games in graph structure
}

Citation for published version (APA):

Herings, P. J. J., van der Laan, G., \& Talman, A. J. J. (2000). Cooperative games in graph structure.

METEOR, Maastricht University School of Business and Economics. METEOR Research Memorandum No. 026 https://doi.org/10.26481/umamet.2000026

Document status and date:

Published: 01/01/2000

DOI:

10.26481/umamet.2000026

Document Version:

Publisher's PDF, also known as Version of record

\section{Please check the document version of this publication:}

- A submitted manuscript is the version of the article upon submission and before peer-review. There can be important differences between the submitted version and the official published version of record.

People interested in the research are advised to contact the author for the final version of the publication, or visit the DOI to the publisher's website.

- The final author version and the galley proof are versions of the publication after peer review.

- The final published version features the final layout of the paper including the volume, issue and page numbers.

Link to publication

\footnotetext{
General rights rights.

- You may freely distribute the URL identifying the publication in the public portal. please follow below link for the End User Agreement:

www.umlib.nl/taverne-license

Take down policy

If you believe that this document breaches copyright please contact us at:

repository@maastrichtuniversity.nl

providing details and we will investigate your claim.
}

Copyright and moral rights for the publications made accessible in the public portal are retained by the authors and/or other copyright owners and it is a condition of accessing publications that users recognise and abide by the legal requirements associated with these

- Users may download and print one copy of any publication from the public portal for the purpose of private study or research.

- You may not further distribute the material or use it for any profit-making activity or commercial gain

If the publication is distributed under the terms of Article $25 \mathrm{fa}$ of the Dutch Copyright Act, indicated by the "Taverne" license above, 


\title{
Cooperative Games in Graph Structure ${ }^{1}$
}

\author{
P. Jean-Jacques Herings ${ }^{2}$ Gerard van der Laan ${ }^{3}$ Dolf Talman ${ }^{4}$
}

August 9, 2000

${ }^{1}$ This research is part of the VF-program "Competition and Cooperation".

${ }^{2}$ P.J.J. Herings, Department of Economics, International Institute for Infonomics, and METEOR, Universiteit Maastricht, P.O. Box 616, 6200 MD Maastricht, The Netherlands, e-mail: p.herings@algec.unimaas.nl

${ }^{3}$ G. van der Laan, Department of Econometrics and Tinbergen Institute, Free University, De Boelelaan 1105, 1081 HV Amsterdam, The Netherlands, e-mail: glaan@econ.vu.nl

${ }^{4}$ A.J.J. Talman, Department of Econometrics and CentER, Tilburg University, P.O. Box 90153, 5000 LE Tilburg, The Netherlands, e-mail: talman@kub.nl 


\begin{abstract}
By a cooperative game in coalitional structure or shortly coalitional game we mean the standard cooperative non-transferable utility game described by a set of payoffs for each coalition that is a nonempty subset of the grand coalition of all players. It is well-known that balancedness is a sufficient condition for the nonemptiness of the core of such a cooperative non-transferable utility game. For this result any information on the internal organization of the coalition is neglected.

In this paper we generalize the concept of coalitional games and allow for organizational structure within coalitions. For a subset of players any arbitrarily given structural relation represented by a graph is allowed for. We then consider non-transferable utility games in which a possibly empty set of payoff vectors is assigned to any graph on every subset of players. Such a game will be called a cooperative game in graph structure or shortly graph game. A payoff vector lies in the core of the game if there is no graph on a group of players which can make all of its members better off.

We define the balanced-core of a graph game as a refinement of the core. To do so, for each graph a power vector is determined that depends on the relative positions of the players within the graph. A collection of graphs will be called balanced if to any graph in the collection a positive weight can be assigned such that the weighted power vectors sum up to the vector of ones. A payoff vector lies in the balanced-core if it lies in the core and the payoff vector is an element of payoff sets of all graphs in some balanced collection of graphs. We prove that any balanced graph game has a nonempty balanced-core and therefore a nonempty core.

We conclude by some examples showing the usefulness of the concepts of graph games and balanced-core. In particular these examples show a close relationship between solutions to noncooperative games and balanced-core elements of a well-defined graph game. This places the paper in the Nash research program, looking for a unifying theory in which each approach helps to justify and clarify the other.
\end{abstract}

Key words: cooperative games, graphs, balancedness, core, Nash program 


\section{Introduction}

Many economic situations can be modelled as a cooperative non-transferable utility game. In such a game a set of payoffs is assigned to each coalition being a subset of the grand coalition. To show nonemptiness of the core of such a game one can use balancedness as a sufficient condition. In the remainder of the paper we will call this condition coalitional balancedness and refer to such a game as a coalitional game. However, in many economic situations of interest more information on the internal organization of a coalition is available. For instance, in order to run a firm, a group of workers can be organised according to several hierarchies consisting of a different number of levels, a different number of workers on a level, or even different players placed on a position of the same internal structure. To each admissible organization of a group of players corresponds a set of payoffs for these players. The usual approach to model this as a cooperative game would be to assign to a coalition of players the union of the sets of payoffs of all possible internal organizations which might occur for that coalition. In this paper we avoid this rough way of modelling and develop tools that exploit the available information about different organizational structures of coalitions.

In the core of a coalitional game it is always assumed that the grand coalition results and cooperates. However, it is not necessarily the case that all players in the grand coalition behave in the same way. The information given in a coalitional game does not reveal that some of the players collude, or one player is the boss over the other players, or that (groups of) players compete with each other. For games with transferable utilities Nowak and Radzik [18] consider games in permutational form. For such TU games the value of a coalition depends on the ordering of the members within the coalition. Myerson [16] uses undirected graphs to model communication structures in cooperative games. Depending on the application it is more appropriate to model the internal organization of a coalition by means of a hierarchy, a partition, an ordered structure as studied in van der Laan, Talman and Yang [14], or an even more general structure. For instance, it may be very useful to model the internal organization of an industry by a partitional game, in which for any coalition of players a payoff set is assigned to every partition of the coalition into subsets of colluding players within the coalition. The obvious question is whether this refined modelling can be utilized to give sharper results on core existence and restrict the core to a smaller set.

To answer this question we generalize the concept of a coalitional game to a graph game. The use of directed graphs provides a very convenient way to model the organizational structure of a set of players. To do so, the nodes of the graph are identified with the players and the arcs of the graph correspond to organizational links between players. Then a cooperative non-transferable utility game in graph structure, or graph game, consists of 
a set of players, a collection of graphs on subsets of the set of all players and a payoff set for every element in the collection of graphs. The whole set of players is called the grand coalition and consists of all players involved in the game. Each graph in the collection of graphs corresponds to a possible internal structure on a coalition of players that could evolve. The collection of possible graphs is called the set of organizational graphs of the game. If, for instance, only hierarchies are relevant, then one could take the collection of all hierarchical graphs on the subsets of players. In the case where partitions are the appropriate model one could take the collection of graphs that have totally connected components. To any organizational graph in the collection of graphs a set of payoffs is assigned reflecting the set of possible outcomes the players can achieve by organizing themselves according to the internal structure given by that graph. So, the set of payoffs attainable to a group of players depends on the organization within that group. It should be stressed that we allow for multiple organizational structures within a given group of players. For every coalition of players, a set of payoffs is assigned to any relational graph structure that might evolve for that coalition.

Following the standard definition for coalitional games, the core of a graph game is defined by the set of all payoff vectors attainable for some internal organization of the whole set of players satisfying that there is no coalition of players that can organize themselves by means of an organizational graph in the collection of possible graphs, such that all players in that coalition can improve upon their payoff.

To prove the nonemptiness of the core we generalize the concept of coalitional balancedness to graph balancedness. To do so, to each graph a power vector is associated whose components reflect the relative position of each player within the graph. Several possibilities to define such power vectors will be discussed. A collection of graphs is defined to be graph balanced if to each graph within the collection a weight can be assigned such that the weighted sum of their power vectors is equal to the vector of ones. A graph game is defined to be balanced if for any balanced collection of graphs the intersection of the corresponding payoff sets is contained in the set of attainable payoff vectors of the grand coalition. A payoff vector is defined to be in the balanced-core of a graph game if it is in the core of the game and it belongs to the intersection of all payoff sets corresponding to some balanced collection of graphs. Obviously the balanced-core of a graph game is a subset of the core. It will be shown that the balanced-core of a balanced graph game is nonempty, by formulating an intersection theorem on the unit simplex that generalizes the well-known intersection theorem of KKM and the generalization that was used by Shapley [21] to prove the nonemptiness of the core of a coalitional game, see also Herings [10], Ichiishi [11] and van der Laan, Talman and Yang [15]. In case the game is a coalitional game the balanced-core equals the core. However, in general the balanced-core of a graph game 
is a proper subset of the core of the game and thus a refinement of the core is obtained.

For any graph game a coalitional game is obtained by defining the payoff set of a coalition as the union over all payoff sets of the possible organizational structures of that coalition. So, in the induced coalitional game all information about the internal organization is ignored. It follows straightforwardly that the core of the induced coalitional game is equal to the core of the underlying graph game, so that graph balancedness of the graph game is sufficient for the non-emptiness of the core of the induced coalitional game. It is well-known that coalitional balancedness guarantees the existence of the nonempty core of the coalitional game. However, graph balancedness of a graph game does not imply coalitional balancedness of the induced coalitional game. From this we may conclude that more information about the internal organizational structure not only refines the core, but also results in less severe conditions for the existence of undominated payoff vectors.

The usefulness of the concept of balanced-core in graph games will be demonstrated by some examples resembling an unexpected relationship between noncooperative and cooperative games. For example, the noncooperative Cournot quantity competition game between firms producing a homogeneous commodity will be reformulated as a cooperative graph game. It will then be shown that the unique element of the balanced-core of the graph game corresponds to the Cournot-Nash equilibrium of the noncooperative game when in the graph game all two-level hierarchical structures of the grand coalition are allowed, while the Cournot-Stackelberg outcome is obtained when only one such a hierarchical graph is allowed, i.e. when the leader is a priori determined. In this way the paper contributes to the Nash research program, see for instance Nash [17], looking for a unifying theory in which each approach helps to justify and clarify the other.

The structure of the paper is as follows. In Section 2 graph games are introduced and several examples of collections of graphs are discussed. We also define the core of a graph game and the coalitional game induced by a graph game. Graph balancedness of a game is introduced in Section 3. To do so, we first introduce the concept of the power vector of a graph. In Section 4 the balanced-core of a graph game is defined and it is proven that the balanced-core is not empty when the graph game is balanced. This also shows the nonemptiness of the core of both the graph balanced game and the induced coalitional game. Some applications and examples are discussed in Section 5. These applications show the relationship between the theories of cooperative and noncooperative games.

\section{Graph games}

Aumann and Peleg [2] have introduced a cooperative non-transferable utility game with a finite number $n$ of players as a game in which for each nonempty subset of players, called 
a coalition, a certain subset of $\mathbb{R}^{n}$ of payoff vectors is available on which the coalition can agree. When a coalition agrees on a payoff vector in the attainable payoff set, then each player of the coalition receives a payoff as specified by this player's component of the payoff vector. A payoff vector that is attainable for the grand coalition lies in the core of the game if no coalition can improve upon this vector, see Aumann [1], i.e. if there does not exist a coalition and a vector in its payoff set that makes at least one player of the coalition better off and gives at least the same payoff to all other players of the coalition. In van der Laan, Talman and Yang [14] this coalitional game is generalized to permutional games in which an attainable set of payoff vectors is assigned to any ordered partition of a coalition of the set of players, i.e. the set of attainable payoff vectors of a coalition is allowed to depend on a given partition of the coalition and a given collection of permutations on the collection of player sets in the partition.

In this paper we further generalize the concept of cooperative non-transferable utility games by allowing that the set of attainable payoff vectors of the players in a coalition depends on the organizational structure within that coalition. To do so, any possible internal structure within a set of players will be modelled by means of a graph. A graph $G$ is defined to be a pair $(V, A(V))$, where $V$ is a non-empty finite set of elements, called the vertices of the graph, and $A(V)$ is a finite collection of ordered pairs of elements of $V$, called the arcs of the graph. An arc is assumed to order always two different vertices. In our setup $V$ is a non-empty subset $S$ of the set $\mathcal{N}=\{1, \ldots, n\}$ of agents or players. ${ }^{1}$ Then a graph $G=(S, A(S))$ denotes a coalition $S \subset \mathcal{N}$ of players and an internal organizational structure within the coalition given by the set $A(S)$ of $\operatorname{arcs}$. For given $A(S)$, any two different agents $i, i^{\prime} \in S$ have no organizational relation in case both pairs $\left(i, i^{\prime}\right)$ and $\left(i^{\prime}, i\right)$ are not in $A(S), i$ is ordered above $i^{\prime}$ if the pair $\left(i, i^{\prime}\right) \in A(S)$, and $i^{\prime}$ is ordered above $i$ if the pair $\left(i^{\prime}, i\right) \in A(S)$. Observe that we don't impose any restrictions on the graph, except that we exclude the pairs $(i, i)$ from $A(S), i \in S$. So, neither completeness nor transitivity of the ordering is required. It should be noticed that we also allow that for some $i, i^{\prime} \in S$, both pairs $\left(i, i^{\prime}\right)$ and $\left(i^{\prime}, i\right)$ are in $A(S)$. To simplify the discussion, an agent $i$ in $S$ is said to dominate $i^{\prime}$ in $S$ within the organizational structure $A(S)$ in case $\left(i, i^{\prime}\right) \in A(S)$ and $\left(i^{\prime}, i\right) \notin A(S)$. Two different agents $i$ and $i^{\prime}$ in $S$ are said to be equally ordered within $A(S)$ in case $A(S)$ contains both $\left(i, i^{\prime}\right)$ and $\left(i^{\prime}, i\right)$. Observe that two equally ordered agents $i$ and $i^{\prime}$ may take different positions within $A(S)$ in the sense that there may exist a third agent $i^{\prime \prime} \in S$ such that $i$ dominates $i^{\prime \prime}$, while $i^{\prime \prime}$ dominates $i^{\prime}$.

For a subset $S$ of $\mathcal{N}$, the collection of all possible graphs $G$ with vertex set $S$ is denoted by $\mathcal{G}(S)$. The collection of all graphs, $\mathcal{G}^{\mathcal{N}}$, is then obtained by taking the union of

\footnotetext{
${ }^{1}$ In the sequel we only consider nonempty subsets $S$ of $\mathcal{N}$.
} 
$\mathcal{G}(S)$ over all $S$, i.e.

$$
\mathcal{G}^{\mathcal{N}}=\cup_{S \subset \mathcal{N}} \mathcal{G}(S)
$$

We now have the following definition of a cooperative graph game, where a set $X \subset \mathbb{R}^{n}$ is said to be cylindric with respect to $S \subset\{1, \ldots, n\}$ if for any two vectors $x$ and $y$ in $\mathbb{R}^{n}$ with $x_{i}=y_{i}$ for all $i \in S$, it holds that $x \in X$ if and only if $y \in X$.

\section{Definition 2.1 (Graph Game)}

A non-transferable utility game in graph structure or graph game on a collection of players $\mathcal{N}=\{1, \ldots, n\}$ consists of a nonempty collection $\Gamma$ of elements of $\mathcal{G}^{\mathcal{N}}$ and a function $v$ from $\Gamma$ to the collection of subsets of $\mathbb{R}^{n}$ (including the empty set) satisfying that for every graph $G \in \Gamma$ being an element of $\mathcal{G}(S)$, the set $v(G) \subset \mathbb{R}^{n}$ is cylindric with respect to $S$.

In the sequel we denote a graph game with a function $v$ on a collection $\Gamma$ of graphs by the pair $(\Gamma, v)$. The collection $\Gamma$ is the set of graphs on coalitions of $\mathcal{N}$ that could evolve in the model and $v$ is the payoff function assigning to every graph in $\Gamma$ a set of payoff vectors. If $u \in v(G)$ with $G \in \mathcal{G}(S)$ for some coalition $S$ of players, then the members of $S$ can guarantee themselves a payoff $u_{i}$ for each $i \in S$, independent of what the players outside the coalition $S$ do, by organizing themselves according to the graph $G$. When $G \in \Gamma$ is a graph in $\mathcal{G}(\mathcal{N})$, then $v(G)$ is the set of payoff vectors the players of the grand coalition $\mathcal{N}$ can guarantee themselves when the players coordinate according to the graph $G$. Before continuing by analyzing the concept of graph games, we first discuss some interesting classes of graph games. To describe these specific collections, we introduce somewhat more terminology.

A graph $(S, A(S))$ is said to be empty if $A(S)=\emptyset$ and is said to be complete if any two different agents $i, i^{\prime} \in S$ are equally ordered, i.e. both $\left(i, i^{\prime}\right)$ and $\left(i^{\prime}, i\right)$ belong to $A(S)$. A complete graph $(S, A(S))$ is also called a coalitional graph. Clearly, for given $S$ the coalitional graph $(S, A(S)$ is uniquely defined and hence the number of coalitional graphs is equal to $2^{n}-1$, namely one graph for each (nonempty) subset $S$ of $\mathcal{N}$. Since in a coalitional graph $(S, A(S))$ each two different players in $S$ are equally ordered, we have that all players are in the same position.

A graph $(S, A(S))$ is said to be connected if it cannot be expressed as the union of two disjoint graphs, i.e. there are no two graphs $G^{1}=\left(S_{1}, A\left(S_{1}\right)\right)$ and $G^{2}=\left(S_{2}, A\left(S_{2}\right)\right)$ such that $S_{1}$ and $S_{2}$ are disjoint, whereas $S=S_{1} \cup S_{2}$ and $A(S)=A\left(S_{1}\right) \cup A\left(S_{2}\right)$. Given a graph $G=(S, A(S))$ on $S$, a path in $G$ is a finite sequence of ordered pairs $\left(i_{1}, i_{2}\right),\left(i_{2}, i_{3}\right), \ldots,\left(i_{m-1}, i_{m}\right)$ such that $i_{j} \in S$ for all $j=1, \ldots, m$ and $\left(i_{j}, i_{j+1}\right) \in A(S)$ for all $j=1, \ldots, m-1$. If $i_{1}=i_{m}$, then the path is called a circuit in $G$. A connected graph 
$G$ without circuits is called a hierarchy. So, a hierarchy only exludes circuits, but does not impose any other restrictions on the graph. The connectness of the graph only states that each two different players in $S$ are connected to each other by a sequence of other players, i.e. in a hierarchical graph $G$ any player $i \in S$ is connected to any other player $i^{\prime} \in S$ by a finite number $m, 0 \leq m \leq|S|-2$, of other players $i_{1}, \ldots, i_{m}$ such that for each $j$, $j=0, \ldots, m$, at least one of the two ordered pairs $\left(i_{j}, i_{j+1}\right)$ and $\left(i_{j+1}, i_{j}\right)$ is in $A(S)$, where $i_{0}=i$ and $i_{m+1}=i^{\prime}$.

In a graph $G=(S, A(S))$, a player $i \in S$ is called a leader or topman if $i$ dominates at least one player $i^{\prime} \in S$, so $\left(i, i^{\prime}\right) \in A(S)$, and there does not exist a player $i^{\prime \prime} \in S$ such that $\left(i^{\prime \prime}, i\right) \in A(S)$, i.e. $i$ is undominated; a player $i \in S$ is called a follower or bottomman if $i$ is dominated by at least one player $i^{\prime} \in S$, so $\left(i^{\prime}, i\right) \in A(S)$, and there does not exist a player $i^{\prime \prime} \in S$ such that $\left(i, i^{\prime \prime}\right) \in A(S)$, i.e. if $i$ does not dominate any other player; a player $i \in S$ is called a middleman if there exist players $i^{\prime}, i^{\prime \prime} \in S$ such that $\left(i, i^{\prime}\right) \in A(S)$ and $\left(i^{\prime \prime}, i\right) \in A(S)$, i.e. if $i$ dominates and is dominated; a player $i \in S$ is called a dummy if for any $i^{\prime} \in S$ neither $\left(i, i^{\prime}\right)$ nor $\left(i^{\prime}, i\right)$ belongs to $S$, i.e. if $i$ dominates nobody and nobody dominates $i$ in $A(S)$. Observe that the sets of topmen, bottommen, middlemen and dummies form a partition of the set $S$.

All players on a circuit in $A(S)$ are middlemen. Since a hierarchical graph is connected, a hierarchy does not contain dummies (except for the trivial hierarchy that consists of one player only. Moreover, a hierarchy has at least one topman and one bottomman, but may contain multiple topmen or bottommen. In the sequel a hierarchy with one topman or one bottomman is called a pseudo-tree. In a pseudo-tree with one topman there is a path from the unique topman to every other player in the graph and in a pseudo-tree with one bottomman there exists for every other player in the hierarchy a path from that player to the unique bottomman. If for every other player the path from the unique topman to that player, respectively from that player to the unique bottomman, is unique, then the pseudo-tree is called a tree. A tree with one topman is called a top-down tree and a tree with one bottomman a bottom-up tree. A tree $(S, A(S))$ with one topman and one bottomman consists of one path from the topman to the bottomman and hence induces an ordering or permutation on the players of $S$. Therefore such a tree is called a permutation tree. For the set $S=\{1,2,3\}$ of three players, all possible types of hierarchies are given in Figure 1. Figure 1a shows a permutation tree with player 1 as the topman, player 2 as middleman and player 3 as the bottomman, Figure $1 \mathrm{~b}$ is a bottom-up tree with players 1 and 2 as topmen and player 3 as the bottomman, and Figure 1c is a top-down tree with player 1 as the topman and the players 2 and 3 as bottommen. Figure $1 \mathrm{~d}$ is a pseudo-tree with player 1 as topman, player 2 as middleman and player 3 as bottomman. Figure 2 shows two types of hierarchies with 2 topmen (1 and 2) and two bottommen (3 and 4). 


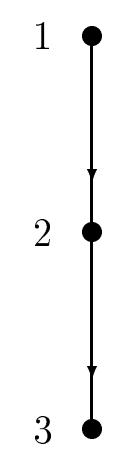

1a

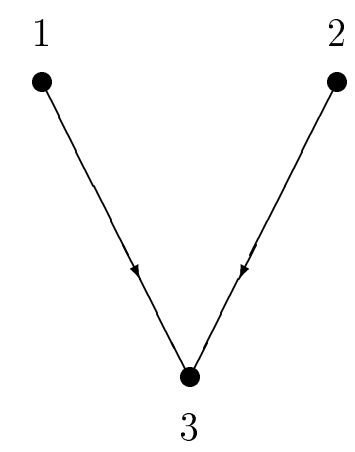

$1 b$

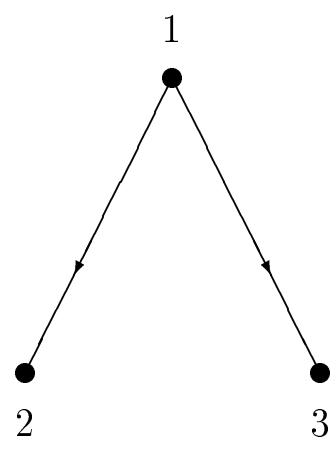

1c

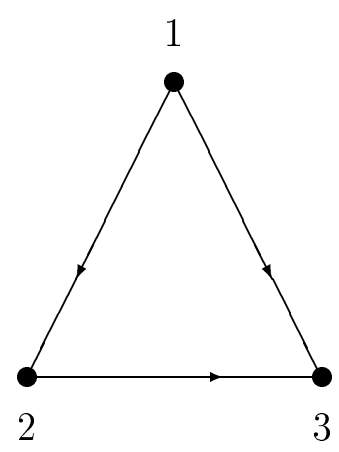

1d

Figure 1. Trees on a three player set.
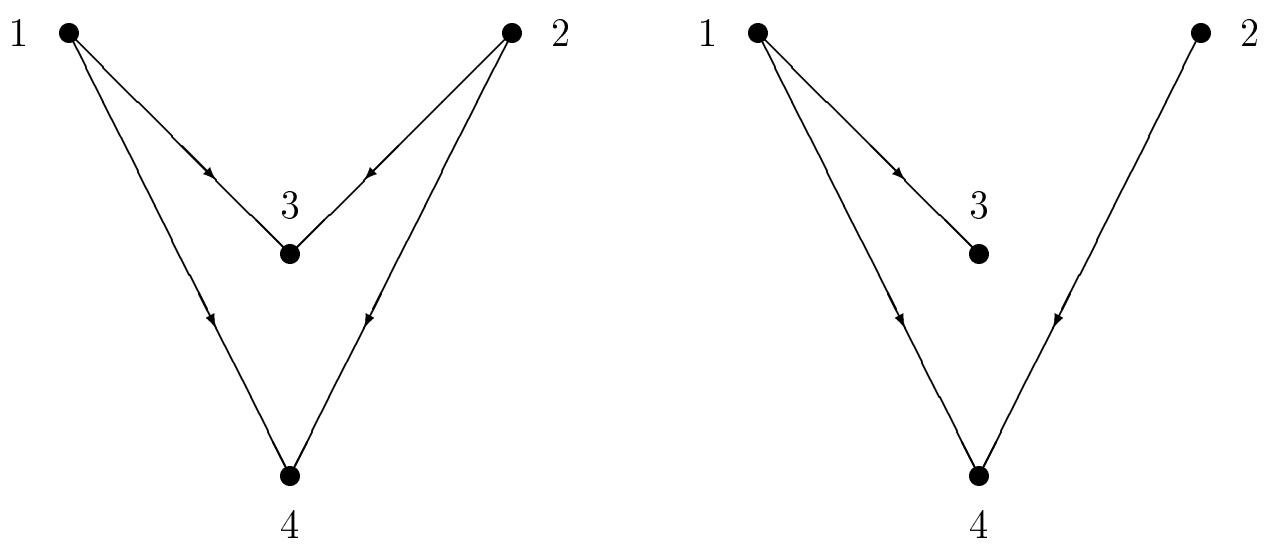

Figure 2. Two hierarchies on a four player set. 


\section{Example 2.2}

We consider the model of the internal organization of a firm as given in Ruys and van den Brink [20]. According to their model a firm is characterized by a top-down tree defined by a number $\ell$ of hierarchical levels and a number $c$ yielding the span of control. The number of hierarchical levels gives the maximal length of a path (the number of arcs) from the unique topman to a bottomman, i.e. each path from the topman to a bottomman passes at most $\ell-1$ middlemen. The span of control gives the number of agents that is dominated by the topman and any of the middlemen, i.e. the topman and each middleman dominate precisely $c$ other agents. For any given set $S$ of players, and any permutation $\left(\pi_{1}, \ldots, \pi_{|S|}\right)$ of the players in $S$, there is a unique permutation tree with $|S|-1$ levels and span of control $c=1$ yielding a path from the topman $\pi_{1}$ to the bottomman $\pi_{|S|}$. Furthermore, for any player $s \in S$ there is a unique top-down tree with 1 level and $c=|S|-1$ having $s$ as the unique topman. Further, for $|S| \leq 4,|S|=6$ and $|S|=8$ there are no other top-down trees with fixed span of control. For $|S|=5$ there is only one other type of top-down tree with fixed span of control, namely with $c=2$ and $\ell=2$. For $|S|=7$ there are three other types of top-down trees with fixed span of control, given in Figure 3, namely trees with $\ell=c=2$, trees with $\ell=2$ and $c=3$ and trees with $\ell=3$ and $c=2$. Observe that the number of trees concerns the number of types, of course many trees are obtained by permutating the players over the different positions in the tree. The set of payoffs may not only differ between different types of trees but may also depend on which players are located at which position on the tree. For example the payoffs for every one might be higher if the more skilled workers are located in the top of the tree and the less skilled workers closer to the bottom.

The example shows that the collection of hierarchical graphs can be severely restricted by making additional assumptions, for instance that the agents can only organize themselves by a top-down tree with constant span of control. As we have seen above, there are for instance only five possible types of trees in case $|S|=7$ and a constant span of control is required. Observe that this number even reduces to the three types, one with $\ell=|S|-1=6$ levels and $c=1$, one with $\ell=1$ level and $c=|S|-1=6$ and one with $c=\ell=2$ when we require that also each path from the topman to a bottomman must have the same length. Another application with a natural limitation on the number of attainable graphs is given in the next example.

\section{Example 2.3}

In this example we consider the organization of an industry with a number of firms competing each by quantity competition. So, each firm first chooses an output quantity and then the price follows from the inverse demand function. A well-known solution for this 

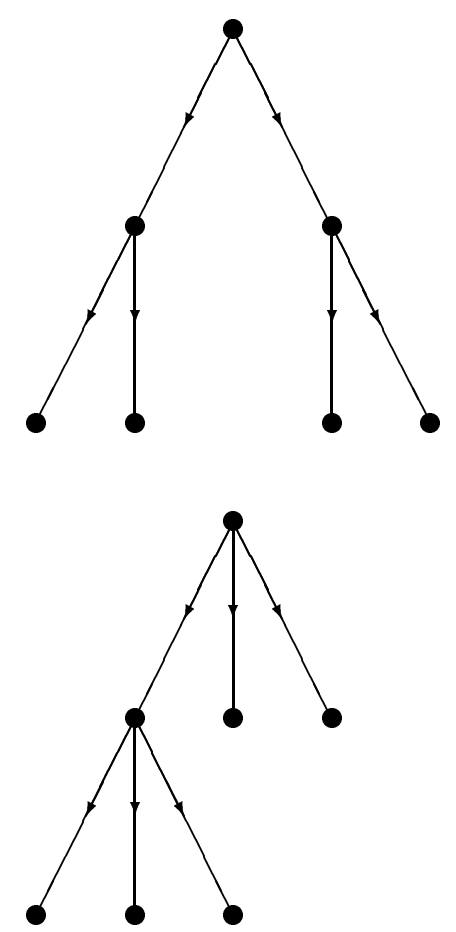

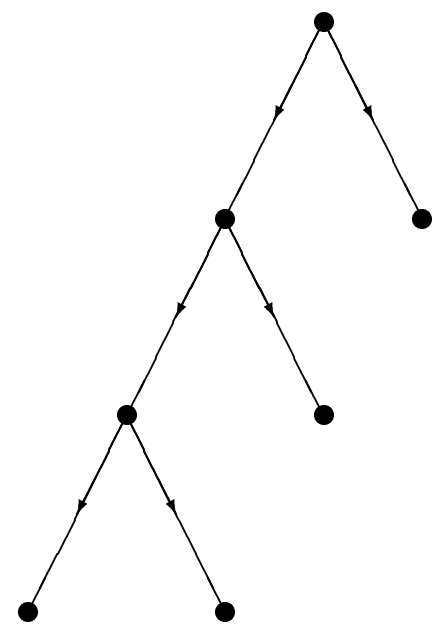

Figure 3. All types of top-down trees with fixed span of control $1<c<6,|S|=7$.

industrial organization model is the Cournot-Nash solution, in which each firm reacts optimally given the behavior of the others, i.e. each firm chooses a profit maximizing quantity of output given the choices of the other firm. In case there are $n$ firms, the industry is modelled by $n$ bottom-up trees with one of the players as bottomman and all other $n-1$ players as topmen. In each tree, first the topmen choose a quantity independent from each other, then the single bottomman reacts optimally on the choices of the topmen.

We now consider some examples of specific graph games. First of all it should be noticed that our general framework comprises the standard coalitional games as a special case.

\section{Definition 2.4 (Coalition game)}

A graph game $(\Gamma, v)$ is a coalitional game if any graph $(S, A(S)) \in \Gamma$ is a coalitional graph. The game $(\Gamma, v)$ is the full coalitional game if $\Gamma$ is the collection of all $2^{n}-1$ coalitional graphs.

A second interesting class of games is the class of partitioned coalitional games. A collection $\left\{\left(S_{1}, A\left(S_{1}\right)\right), \ldots,\left(S_{m}, A\left(S_{m}\right)\right)\right\}$ of $m$ graphs is a partition of the graph $(S, A(S))$ if the collection $\left\{S_{1}, \ldots, S_{m}\right\}$ is a partition of the set $S$ of vertices and the collection $\left\{A\left(S_{1}\right), \ldots, A\left(S_{m}\right)\right\}$ is a partition of the set $A(S)$ of arcs. 


\section{Definition 2.5 (Partitioned Coalition game)}

A graph game $(\Gamma, v)$ is a partitioned coalitional game if every graph $(S, A(S)) \in \Gamma$ can be partitioned into a collection $\left\{\left(S_{1}, A\left(S_{1}\right)\right), \ldots,\left(S_{m}, A\left(S_{m}\right)\right)\right\}$ of coalitional graphs.

Observe that for any given $S$ and any given partition $\left\{S_{1}, \ldots, S_{m}\right\}$ of $S$ the graph $(S, A(S))$ such that $\left\{\left(S_{1}, A\left(S_{1}\right)\right), \ldots,\left(S_{m}, A\left(S_{m}\right)\right)\right\}$ is a partition of $(S, A(S))$ into coalitional graphs is uniquely defined, namely the graph $(S, A(S))$ being the union of the collection of the coalitional graphs $\left(S_{j}, A\left(S_{j}\right)\right), j=1, \ldots, m$. In van der Laan, Talman and Yang [14] cooperative games have been studied which can be modelled within the framework of this paper by the collection of ordered partitioned coalitional graphs. A graph $(S, A(S))$ is an ordered partitioned coalitional graph if the set $S$ can be partitioned in a collection of subsets $S_{j}, j=1, \ldots, m$, such that for any two players $i, i^{\prime} \in S$ it holds that $\left(i, i^{\prime}\right) \in A(S)$ if and only if either for some $j=1, \ldots, m$, both $i$ and $i^{\prime}$ belong to the same subset $S_{j}$, or for some $j=1, \ldots, m-1$, we have that $i \in S_{j}$ and $i^{\prime} \in S_{j+1}$, i.e. for all $j=1, \ldots, m$ the graph restricted to $S_{j}$ is a coalitional graph and the partition $\left\{S_{1}, \ldots, S_{m}\right\}$ if $S$ is ordered in the sense that any player in the set $S_{j}$ dominates any player in $S_{j+1}$ or shortly $S_{j}$ dominates $S_{j+1}, j=1, \ldots, m-1$.

\section{Definition 2.6 (Ordered Partitioned Coalition game)}

A graph game $(\Gamma, v)$ is an ordered partitioned coalition game if every graph $(S, A(S)) \in \Gamma$ is an ordered partitioned coalitional graph.

Finally we define hierarchy games and some subclasses of hierachy games.

\section{Definition 2.7 (Hierarchy, Tree and Permutation games)}

A graph game $(\Gamma, v)$ is a hierarchy game if every graph $(S, A(S)) \in \Gamma$ is a hierarchy. A hierarchy game $(\Gamma, v)$ is a tree game if every graph $(S, A(S)) \in \Gamma$ is a tree.

A tree game $(\Gamma, v)$ is a permutation game if every graph $(S, A(S)) \in \Gamma$ is a permutation tree.

It should be noticed that the analysis in this paper is entirely valid in the most general case where no a priori restrictions are placed on the internal structures of coalitions, i.e. in case $\Gamma=\mathcal{G}^{\mathcal{N}}$. However, restricting the graphs to a certain subclass of the class $\mathcal{G}^{\mathcal{N}}$ of all graphs and hence reducing the complexity of the game to a game $(\Gamma, v)$ for some subset $\Gamma$ of $\mathcal{G}^{\mathcal{N}}$ satisfying certain properties will often facilitate the analysis. The possibility to do so of course depends on the underlying application.

We conclude this section by restating the core concept for graph games and the relationship between the core of a graph game and the core of a coalitional game induced 
by the graph game. For any graph game $(\Gamma, v)$, let the function $v^{\prime}$ from the collection of subsets of $\mathcal{N}$ to the collection of subsets of $\mathbb{R}^{n}$ be defined by

$$
v^{\prime}(S)=\cup_{G \in \Gamma \cap \mathcal{G}(S)} v(G), S \subset \mathcal{N}
$$

i.e. the payoff set $v^{\prime}(S)$ is defined to be the union of all payoff sets assigned to the graphs on the coalition $S$ of players. This function $v^{\prime}$ induces a non-transferable utility $n$-player game in coalitional structure, denoted by $\left(\mathcal{N}, v^{\prime}\right)$. Since $\Gamma \subset \mathcal{G}^{\mathcal{N}}$ and we also allow for empty payoff sets if $G \in \Gamma$, it might be possible that also some of the payoff sets $v^{\prime}(S)$ are empty. The core of the induced coalitional game $\left(\mathcal{N}, v^{\prime}\right)$, denoted by $C\left(\mathcal{N}, v^{\prime}\right)$, is as usual defined by the set of vectors $x \in v^{\prime}(\mathcal{N})$ such that there do not exist a coalition $S \subset \mathcal{N}$ and vector $y \in v^{\prime}(S)$, such that $y_{i}>x_{i}$ for all $i \in S$. Analogously we say that a payoff vector $x$ is in the core of the graph game $(\Gamma, v)$ if $x \in v^{\prime}(\mathcal{N})=\cup_{G \in \Gamma \cap \mathcal{G}(\mathcal{N})} v(G)$ and there are no coalition $S$ and graph $G \in \Gamma \cap \mathcal{G}(S)$, such that all the members of the coalition $S$ can improve upon $x$.

\section{Definition 2.8 (Core of a Graph Game)}

The core of a non-transferable utility graph game $(\Gamma, v)$ is the set of vectors $x \in \mathbb{R}^{n}$ satisfying that $x \in v^{\prime}(\mathcal{N})$ and there do not exist a coalition $S \subset \mathcal{N}$, graph $G \in \Gamma \cap \mathcal{G}(S)$ and vector $y \in v(G)$ such that $y_{i}>x_{i}$ for all $i \in S$.

In the sequel we denote the core of a graph game $(\Gamma, v)$ by $C(\Gamma, v)$. Observe that a core element of the graph game lies in $v(G)$ of some graph $G \in \mathcal{G}(\mathcal{N})$ on the grand coalition and hence lies in $v^{\prime}(\mathcal{N})$. Now we have the following lemma, which shows that the core of a graph game coincides with the core of the induced coalitional game.

\section{Lemma 2.9}

For any graph game $(\Gamma, v)$ and the induced coalitional game $\left(\mathcal{N}, v^{\prime}\right)$ it holds that $C(\Gamma, v)=$ $C\left(\mathcal{N}, v^{\prime}\right)$.

\section{Proof.}

For some $x \in \mathbb{R}^{n}$, first suppose $x \notin C\left(\mathcal{N}, v^{\prime}\right)$. Then there exists a coalition $S \subset \mathcal{N}$ and a vector $y \in \mathbb{R}^{n}$ such that $y \in v^{\prime}(S)$ and $y_{i}>x_{i}$ for all $i \in S$. By the definition of $v^{\prime}(S)$ this implies that there is some graph $G$ in $\Gamma \cap \mathcal{G}(S)$ such that $y \in v(G)$. Hence $x \notin C(\Gamma, v)$. Secondly, suppose that $x \notin C(\Gamma, v)$. Then there exist a coalition $S$, graph $G \in \Gamma \cap \mathcal{G}(S)$ and vector $y \in v(G)$ such that $y_{i}>x_{i}$ for all $i \in S$. By definition we have that $y \in v^{\prime}(S)$. Hence $x \notin C\left(\mathcal{N}, v^{\prime}\right)$.

Q.E.D. 


\section{Balancedness of graph games}

It is well-known that the core of a non-transferable utility coalitional game is not empty if the game is balanced. In this section we will generalize the concept of balancedness for coalitional games to balancedness for graph games. Based on this definition we introduce the concept of balanced-core of a graph game and show that an element in the balancedcore is also in the core of the graph game. In the next section we prove that a balanced graph game has a non-empty balanced-core.

First we introduce the notion of power measure on a graph. For given $S \subset \mathcal{N}$, let $G_{S}=(S, A(S))$ be a graph in $\mathcal{G}(S)$. Then a power measure on $G_{S}$ assigns a real number to any player in $S$, which can be seen as the importance or power of that player within the graph $G_{S}$. To facilitate the introduction of power measure, we first define for every player $i \in S$ in a graph $G_{S}$ the sets of predecessors and successors by

$$
P^{i}\left(G_{S}\right)=\{j \in S \mid(j, i) \in A(S)\}
$$

and

$$
D^{i}\left(G_{S}\right)=\{j \in S \mid(i, j) \in A(S)\}
$$

respectively, i.e. $P^{i}\left(G_{S}\right)$ is the set of all players by which $i$ is dominated in $G_{S}$ and $D^{i}\left(G_{S}\right)$ is the set of all players in $G_{S}$ dominated by $i$. A power measure that is well-known in graph theory is the score measure, see for instance Behzad, Chartrand and Lesniak-Foster [3] and van den Brink [6]. For tournaments, i.e. graphs $(S, A(S))$ such that for any pair $i, i^{\prime}$ of two different players in $S$ either $\left(i, i^{\prime}\right)$ or $\left(i^{\prime}, i\right)$ is in $A(S)$ the score measure coincides with the Copeland measure and has been axiomatized by Rubinstein [19], see also Bouyssou [5]. According to the score measure, the power of a player $i$ in $G_{S}$ is equal to the number of elements in the set $D^{i}\left(G_{S}\right)$. Another measure has been developed by van den Brink and Gilles [7], see also [8], according to which the power of a player $i$ in $G_{S}$ is given by $\sum_{j \in D^{i}\left(G_{S}\right)}\left|P^{j}\left(G_{S}\right)\right|^{-1}$. The interpretation of this so-called BG or dominance measure is as follows. Initially, each player gets one point. This point is equally distributed amongst all his predecessors, so amongst all the players by which a player is dominated. The power of a player is the sum of all his shares in the points of the players he dominates.

Clearly, except when $A(S)$ is empty, each of the above mentioned measures can be easily normalized in such a way that the sum of the powers is equal to one. When $A(S)$ is empty, it is natural to set the normalized power of a player in $G_{S}$ equal to $|S|^{-1}$. For our purposes, we further assign for each $G_{S}$ also a power to all players not in $S$ by setting the power of a player $j$ equal to zero when $j \notin S$. This gives the following definition of a power measure and a power measure function, a function that associates a power measure to each graph in $\mathcal{G}^{\mathcal{N}}$. 


\section{Definition 3.1 Power measure}

Let $S \subset \mathcal{N}$ be a coalition of players and $G_{S}=(S, A(S))$ a graph on $S$. Then a power measure for $G_{S}$ is a vector $m\left(G_{S}\right) \in \mathbb{R}^{n}$ such that

(i) $m_{j}\left(G_{S}\right)=0$ if $j \notin S$,

(ii) $\quad \sum_{i \in S} m_{i}\left(G_{S}\right)=1$.

A power measure function for $\mathcal{G}^{\mathcal{N}}$ is a function $m: \mathcal{G}^{\mathcal{N}} \rightarrow \mathbb{R}^{n}$ such that $m\left(G_{S}\right)$ is a power measure for all $G_{S} \in \mathcal{G}^{\mathcal{N}}$.

For practical use the above definition of power measure does not require enough to be useful in particular situations. For example, the vector $\widehat{m}\left(G_{S}\right)$ defined by $\widehat{m}_{i}\left(G_{S}\right)=1 /|S|$ for all $i \in S$ and $\widehat{m}_{i}\left(G_{S}\right)=0$ for all $i \notin S$ satisfies the conditions. In this case, the power measure depends on $S$, but not on the graph $G_{S}$ on $S$. In fact this measure is usually applied in coalitional games to define balancedness. In our more general framework of graph games, we want to allow for a graph dependent power measure. This does not necessarily mean that two different graphs always get different power measures. For instance, it is quite natural to assign the above defined vector $\widehat{m}$ for both the empty graph and the coalitional graph on a set $S$, and also for any other graph in which all players take symmetric positions, e.g. the circuit given by the graph $G_{S}=(S, A(S))$ with $A(S)=\left\{\left(i_{j}, i_{j+1}\right)|j=1, \ldots| S \mid,\right\}$, where $i_{1}, i_{2}, \ldots, i_{|S|}$ are the different players in $S$ and $i_{|S|+1}=i_{1}$. On the other hand, it is natural to allow that players have different power when they do not take symmetric positions in the graph. Therefore we introduce a power measure that will be called the positional measure. While the score measure of a player only depends on his number of successors and the dominance measure of a player on his set of successors and the sets of predecessors of all his successors, the positional measure of a player in a graph $G_{S}=(S, A(S))$ depends on all arcs in the set $A(S)$, i.e. it depends on the full graph and the position of the player within the graph.

To define the positional measure we consider the system of $|S|$ equations induced by a graph $G_{S}=(S, A(S))$ given by

$$
x_{i}=c+\sum_{j \in D^{i}\left(G_{S}\right)} x_{j}-\sum_{j \in P^{i}\left(G_{S}\right)} x_{j}, i \in S,
$$

where $c \in \mathbb{R}$ is a constant to be determined below. Bringing the two last righthand side terms to the lefthand side of the equation, this system of equations can be written as

$$
M\left(G_{S}\right) \cdot x=c e^{S},
$$

where $x$ is the $|S|$-dimensional vector with components $\left(x_{i}\right)_{i \in S}$ and $e^{S}$ the $|S|$-dimensional vector of ones, and where $M\left(G_{S}\right)$ is the $|S| \times|S|$ matrix given by

$$
M_{i i}\left(G_{S}\right)=1 \text { for all } i \in S
$$


and for all $i \neq j, i, j \in S$ by

$$
\begin{aligned}
& M_{i j}\left(G_{S}\right)=1 \text { if } j \in P^{i}\left(G_{S}\right) \text { and } j \notin D^{i}\left(G_{S}\right), \\
& M_{i j}\left(G_{S}\right)=-1 \text { if } j \in D^{i}\left(G_{S}\right) \text { and } j \notin P^{i}\left(G_{S}\right), \\
& M_{i j}\left(G_{S}\right)=0 \text { otherwise. }
\end{aligned}
$$

For any graph $G_{S}$, we now have the following lemma on the properties of the matrix $M\left(G_{S}\right)$, where $I^{S}$ the $|S| \times|S|$ identity matrix and $0^{S}$ the $|S|$-dimensional vector of zeros.

\section{Lemma 3.2}

For any graph $G_{S} \in \mathcal{G}^{\mathcal{N}}$ it holds that

$$
M\left(G_{S}\right)+M^{\top}\left(G_{S}\right)=2 I^{S}
$$

(ii) $\quad x^{\top} M\left(G_{S}\right) x=\sum_{i=1}^{|S|}\left(x_{i}\right)^{2}$ for any $x=\left(x_{i}\right)_{i \in S}$,

(iii) $M^{-1}\left(G_{S}\right)$ exists.

\section{Proof.}

(i) For all $i \neq j$ we have that $M_{i j}\left(G_{S}\right)=0$ if and only if $M_{j i}\left(G_{S}\right)=0$ and $M_{i j}\left(G_{S}\right)=1$ if and only if $M_{j i}\left(G_{S}\right)=-1$. Together with $M_{i i}\left(G_{S}\right)=1$ for all $i$, it follows that $M\left(G_{S}\right)+M^{\top}\left(G_{S}\right)=2 I^{S}$.

(ii) From (i) it follows that for any $x=\left(x_{i}\right)_{i \in S}$ it holds that $x^{\top} M\left(G_{S}\right) x=\frac{1}{2} x^{\top}\left(M\left(G_{S}\right)+\right.$ $\left.M^{\top}\left(G_{S}\right)\right) x=x^{\top} I x=\sum_{i=1}^{|S|}\left(x_{i}\right)^{2}$.

(iii) From (ii) it follows that $x^{\top} M\left(G_{S}\right) x=\sum_{i=1}^{|S|}\left(x_{i}\right)^{2}>0$ if $x \neq 0^{S}$. Hence, $M\left(G_{S}\right)$ is regular, so that $M^{-1}\left(G_{S}\right)$ exists.

Q.E.D.

From part (iii) of the lemma it follows that for any graph $G_{S}$ and any $c$ the system (2) has a unique solution $x\left(G_{S}, c\right)$ given by the vector

$$
x\left(G_{S}, c\right)=c M^{-1}\left(G_{S}\right) e^{S} .
$$

Clearly, $x\left(G_{S}, c\right)=c x\left(G_{S}, 1\right)$. Moreover, it follows that

$$
\sum_{i=1}^{|S|} x_{i}\left(G_{S}, c\right)=e^{S^{\top}} x\left(G_{S}, c\right)=c e^{S^{\top}} M^{-1}\left(G_{S}\right) e^{S} .
$$

So, taking $c^{*}=\left(e^{S^{\top}} M^{-1}\left(G_{S}\right) e^{S}\right)^{-1}$ and defining $x\left(G_{S}\right)=x\left(G_{S}, c^{*}\right)=c^{*} M^{-1}\left(G_{S}\right) e^{S}$, it follows that

$$
\sum_{i=1}^{|S|} x_{i}\left(G_{S}\right)=c^{*} e^{S^{\top}} M^{-1}\left(G_{S}\right) e^{S}=1 .
$$

We now define the positional measure of a player $i$ in a graph $G_{S}$ as $x_{i}\left(G_{S}\right)$ if $i \in S$ and zero otherwise. 


\section{Definition 3.3 Positional measure}

Let $S \subset \mathcal{N}$ be a coalition of players with organizational structure $G_{S} \in \mathcal{G}(S)$. Then the positional measure is the vector $m^{p}\left(G_{S}\right) \in \mathbb{R}^{n}$ given by

$$
\begin{aligned}
& m_{i}^{p}\left(G_{S}\right)=0 \text { if } i \notin S, \\
& m_{i}^{p}\left(G_{S}\right)=x_{i}\left(G_{S}\right) \text { if } i \in S .
\end{aligned}
$$

By definition we have that the sum of the components of the power measure is equal to one. So, according to system (1) the positional power $x_{i}\left(G_{S}\right)$ of a player $i \in S$ is equal to the normalization constant $c^{*}=\left(e^{S^{\top}} M^{-1}\left(G_{S}\right) e^{S}\right)^{-1}$ plus the sum of the powers of all his successors (all players dominated by $i$ ) minus the sum of the powers of all his predecessors (all players which dominate $i$ ).

In the next examples the normalized score measure, denoted by $m^{s}\left(G_{S}\right)$, the normalized dominance measure, denoted by $m^{d}\left(G_{S}\right)$ and the positional measure $m^{p}\left(G_{S}\right)$ are compared with each other for some typical graphs $G_{S}$. To avoid notational burden, in all examples we take $S=\mathcal{N}$.

\section{Example 3.4}

First, let $S=\{1, \ldots, n\}$ and let $G_{S}$ be the coalitional graph, the empty graph or a circuit, respectively. In all three cases we have that $m_{i}^{p}\left(G_{S}\right)=m_{i}^{s}\left(G_{S}\right)=m_{i}^{d}\left(G_{S}\right)=\frac{1}{n}$ for all $i=1, \ldots, n$.

Second, we consider the five player graph on the set $S=\{1, \ldots, 5\}$ of players being partitioned in the coalitional graph on the set $S_{1}=\{1,2,3\}$ and the coalitional graph on the set $S_{2}=\{4,5\}$. The measures for this graph are given by:

$$
\begin{aligned}
m^{s}\left(G_{S}\right) & =\frac{1}{8}(2,2,2,1,1)^{\top}, \\
m^{d}\left(G_{S}\right) & =\frac{1}{5}(1,1,1,1,1)^{\top}, \\
m^{p}\left(G_{S}\right) & =\frac{1}{5}(1,1,1,1,1)^{\top} .
\end{aligned}
$$

In this case only the score measure differentiates between the different positions taken by the first three players and the last two players.

\section{Example 3.5}

Let $n=4$ and let $G_{S}$ be the permutation tree on $S$ given by $A(S)=\{(i, i+1) \mid i=1,2,3\}$. Then the measures are as follows:

$$
\begin{aligned}
m^{s}\left(G_{S}\right) & =\frac{1}{3}(1,1,1,0)^{\top}, \\
m^{d}\left(G_{S}\right) & =\frac{1}{3}(1,1,1,0)^{\top}, \\
m^{p}\left(G_{S}\right) & =\frac{1}{14}(7,2,4,1)^{\top} .
\end{aligned}
$$


The first two measures don't differentiate between the topman 1 and the two middlemen 2 and 3. The positional measure differentiates between the topman and the other players. These properties hold for any permutation tree. In this example we also see that the positional measure gives the topman the highest power and the bottomman the lowest. The former property is true for any permutation tree, but the latter is not. For a permutation tree the positional measure typically shows that the powers have alternating high and low values. The high power of the topman induces a low power for the second man. According to equation (1) this results in a higher power for the third man in the hierarchy and so on. For $n=3$ the positional measure is given by $m^{p}\left(G_{S}\right)=\frac{1}{7}(4,1,2)^{\top}$. In some organizations this maybe indeed reflect the weak position of the middlemen as intermediator between the topman and the bottomman. Since the middleman gets his direct orders from the powerful topman, his power is quite limited. The bottomman, dominated by the weak middleman, may have more possibilities for making his own decisions. For higher values of $n$ the alternating scheme dies out when getting lower in the tree, for instance for $n=9$ the positional measure is given by $m^{p}\left(G_{S}\right)=\frac{1}{39} \cdot(8,3,6,4,5,4,4,3,2)^{\top}$.

\section{Example 3.6}

We now consider the trees given in Figure 4, where the indices of the players are indicated in the figure. The respective measures are:

$$
\begin{array}{llll} 
& \text { graph 4a } & \text { graph 4b } & \text { graph 4c } \\
m^{s}\left(G_{S}\right) & \frac{1}{3}(1,1,1,0,0,0,0)^{\top} & \frac{1}{2}(1,1,0,0,0)^{\top} & \frac{1}{3}(1,2,0,0)^{\top}, \\
m^{d}\left(G_{S}\right) & \frac{1}{3}(1,1,1,0,0,0,0)^{\top} & \frac{1}{2}(1,1,0,0,0)^{\top} & \frac{1}{4}(1,3,0,0)^{\top}, \\
m^{p}\left(G_{S}\right) & \frac{1}{25}(9,2,2,3,3,3,3)^{\top} & \frac{1}{17}(9,4,-2,3,3)^{\top} & \frac{1}{6}(3,4,-2,1)^{\top} .
\end{array}
$$

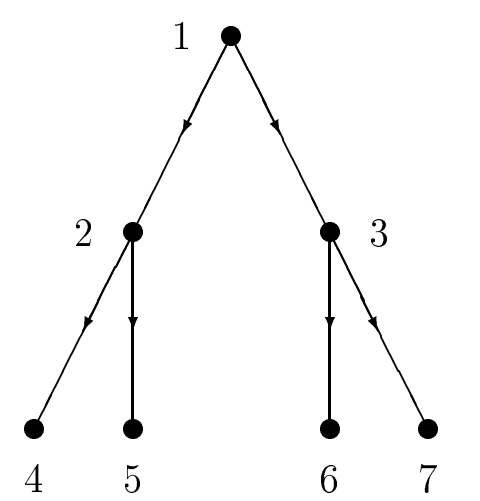

$4 a$

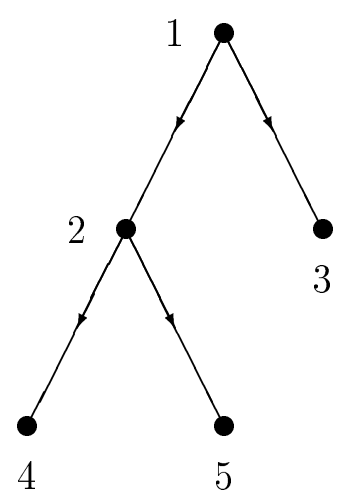

$4 \mathrm{~b}$

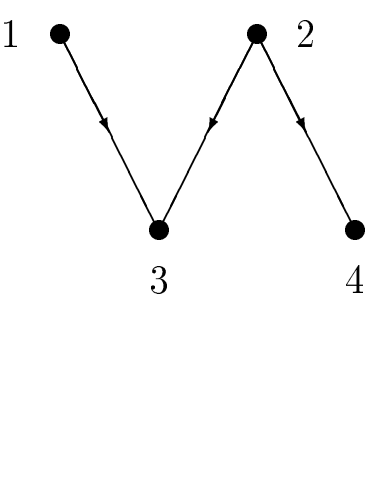

4c

Figure 4. 
For the 7 player top-down tree with fixed span of control of Figure 4a we see again that in the positional measure the middlemen have the lowest power. In the 5 player top-down tree of Figure $4 \mathrm{~b}$ the positional measure gives the player 3, dominated by the strong topman and not dominating any player himself, a negative power. In both cases the score measure is equal to the dominance measure, which is due to the fact that each player is dominated by at most one other player. In the four player hierarchy of Figure 4c player 3 is dominated by both 1 and 2, while player 4 is dominated by player 2 only. In this case the score measure and the dominance measure are different. Observe that in the latter example the positional measure not only differentiates between the two top players, but also differentiates between the two bottom players 3 and 4, according to the different positions in the graph taken by these two players. Because of the weak position of player 3, the positional power of this player becomes negative.

\section{Example 3.7}

Finally we consider the three player graphs given in Figure 5. In these graphs the set of players $S=\{1,2,3\}$ is partitioned into the collection $S_{1}=\{1\}$ and $S_{2}=\{2,3\}$. Figure $5 \mathrm{a}$ is a partitioned graph with a permutation tree on the set $S_{2}$. The graphs of Figures $5 \mathrm{~b}$ and $5 \mathrm{c}$ are ordered coalitional graphs. In $5 \mathrm{~b} S_{1}$ dominates $S_{2}$ and in $5 \mathrm{c} S_{2}$ dominates $S_{1}$. The respective measures are:

$$
\begin{array}{llll} 
& \text { graph 5a } & \text { graph 5b } & \text { graph 5c } \\
m^{s}\left(G_{S}\right) & (0,1,0)^{\top} & \frac{1}{4}(2,1,1)^{\top} & \frac{1}{2}(0,1,1)^{\top}, \\
m^{d}\left(G_{S}\right) & (0,1,0)^{\top} & \frac{1}{4}(2,1,1)^{\top} & \frac{1}{2}(0,1,1)^{\top}, \\
m^{p}\left(G_{S}\right) & \frac{1}{2}(1,1,0)^{\top} & (1,0,0)^{\top} & (-1,1,1)^{\top} .
\end{array}
$$
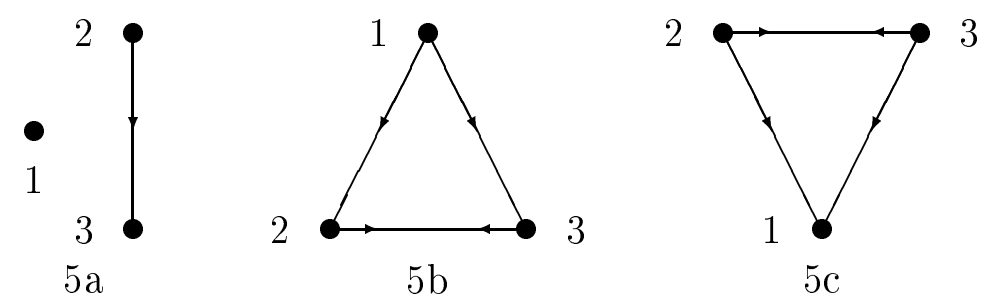

Figure 5 .

The most appropriate measure to use will depend on the particular application one has in mind. For instance, as we have seen in Example 3.4 the dominance measure and the 
positional measure do not discriminate between players in a partitioned coalitional graph. So, when considering an example in which all available graphs are coalitional partitions, there are cases in which one wants to take the normalized score measure as the most appropriate measure, giving more power to a player when the number of players in the subset to which that player belongs increases. On the other hand, being part of a larger coalition means also to coordinate with more players, which has an offsetting effect on the power of an individual player, and which makes the positional measure more appropriate.

We now have the following definition of graph balancedness, where $m$ is some power measure as defined in Definition 3.1. It should be observed that the definition depends on the chosen power measure, i.e. a family of graphs may be balanced for some power measure and not for some other measure. Nevertheless, the following analysis is valid for any power measure.

\section{Definition 3.8 (Graph Balancedness)}

For a given power measure function $m$, a family $\mathcal{F}=\left\{F^{1}, \ldots, F^{k}\right\}$ of $k$ graphs in the collection $\mathcal{G}^{\mathcal{N}}$ is graph balanced if there exist positive numbers $\lambda_{j}, j=1, \ldots, k$, such that

$$
\sum_{j=1}^{k} \lambda_{j} m\left(F^{j}\right)=\frac{1}{n} e^{\mathcal{N}} .
$$

Graph balancedness of a family $\mathcal{F}$ of $k$ graphs $F^{1}, \ldots, F^{k}$ in $\mathcal{G}^{\mathcal{N}}$ means that to any graph $F^{j}, j=1, \ldots, k$, a weight $\lambda_{j}$ can be assigned in such a way that the total power of every player $i \in \mathcal{N}$ is the same and therefore is equal to $\frac{1}{n}$. Geometrically it means that $\mathcal{F}$ is graph balanced if and only if the vector $\frac{1}{n} e^{\mathcal{N}}$ lies in the relative interior of the convex hull of the vectors $m\left(F^{j}\right), j=1, \ldots, k$. Notice that in Definition 3.8 it must

hold that $\sum_{j=1}^{k} \lambda_{j}=1$. Observe that graph balancedness coincides with the well-known notion of coalitional balancedness when every graph $F^{k}=\left(S^{k}, A\left(S^{k}\right)\right)$ in the family $\mathcal{F}$ is a coalitional graph and the power measure satisfies $m_{j}\left(F^{k}\right)=1 /\left|S^{k}\right|$ if $j \in S^{k}$ and $m_{j}\left(F^{k}\right)=0$ otherwise. We now have the following definition of a balanced graph game.

\section{Definition 3.9 (Balanced graph game)}

A non-transferable utility game $(\Gamma, v)$ with power measure function $m$ is a balanced graph game if for every graph balanced family $\mathcal{F}=\left\{F^{1}, \ldots, F^{k}\right\}$ of graphs in $\Gamma$ it holds that

$$
\cap_{j=1}^{k} v\left(F^{j}\right) \subset v^{\prime}(\mathcal{N}) \text {. }
$$

Observe that this definition boils down to the standard definition of a balanced coalitional game when we take $\Gamma$ as the collection of all coalitional graphs. In the sequel we speak shortly about a balanced graph game (balanced coalitional game), if we mean a graph balanced (coalitionally balanced), non-transferable utility graph game (coalitional game 
respectively). Surprisingly, the balancedness of a graph game does not imply that also the induced coalitional game as defined in the previous section is a balanced coalitional game. For an example where $\Gamma$ is the collection of all ordered coalitional partitions, we refer to van der Laan, Talman and Yang [14].

In the next section it will be shown that the core is nonempty if the graph game is graph balanced and every set $v(G), G \in \Gamma \cap \mathcal{G}(S)$, is comprehensive, closed and bounded from above in its projection space $\mathbb{R}^{S}$ defined by $\mathbb{R}^{S}=\left\{\left(x_{i}\right)_{i \in S} \mid x \in \mathbb{R}^{n}\right\}$. From Lemma 2.9 it also follows that it is sufficient for the nonemptiness of the core of a coalitional game induced by a graph game that the underlying graph game is balanced and hence it is not required that the coalitional game itself is coalitionally balanced.

To conclude this section we remark that Billera [4] has pointed out that in case of coalitional games there are many ways to define the powers of the players. In the same way there is a lot of freedom to define the powers in case of graph games. Notice however that this has consequences in forming graph balanced families and hence on the fact whether or not a game in graph structure is graph balanced. Since the core of a game does not depend on the definition of the power vectors, this implies that for the nonemptiness of the core of a graph game it is sufficient to have graph balancedness with respect to some

collection of power vectors. For example, if we take $m(G)=\frac{1}{|S|} e^{S}$ for every $G \in \Gamma \cap \mathcal{G}(S)$, then the graph game is balanced with respect to these constant (for every $S$ ) vectors if and only if the induced coalitional game is balanced. Hence, an induced coalitionally game being balanced implies that the original graph game is graph balanced with respect to some specific collection of power vectors. However, the other way around is not true, i.e. a coalitional game induced by a balanced graph game may not be coalitionally balanced with respect to any set of power vectors. Moreover, while the core is independent of the choice of the power vectors, the balanced-core to be introduced in the next section will depend on this choice. The choice of the power vectors should depend on the economic, or in general game theoretic, situation under consideration. The appropriate choice of power vectors in a given application is a point of further research.

\section{Balanced-core}

For graph games the concept of the core can be refined to what we will call the balancedcore. The balanced-core of a graph game consists of all elements of the core that can be supported by a balanced collection of graphs of coalitions and will be denoted by $B C(\Gamma, v)$. We show that the balanced-core of the graph game $(\Gamma, v)$ is nonempty if the game is graph balanced. Furthermore, from the definition of the balanced-core it follows immediately that the balanced-core of a graph game is a subset of the core of this game, and in many 
cases a proper subset of the core. In case $\Gamma$ is the collection of all coalitional games and so $(\Gamma, v)$ is a coalitional game, then its balanced-core is equal to the usual core of a coalitional game.

\section{Definition 4.1 (Balanced-core)}

The balanced-core of a non-transferable utility graph game $(\Gamma, v)$ with power measure function $m$ is the set of all vectors $x \in \cup_{G \in \Gamma \cap \mathcal{G}(\mathcal{N})} v(G)$ satisfying that

(i) for any $S \subset \mathcal{N}$, there does not exist a graph $G \in \Gamma \cap \mathcal{G}(S)$ and a vector $y \in v(G)$ such that $y_{i}>x_{i}$ for all $i \in S$,

(ii) there exists a graph balanced family $\left\{F^{1}, \ldots, F^{k}\right\}$ of $k$ graphs in $\Gamma$, such that $x \in$ $\cap_{j=1}^{k} v\left(F^{j}\right)$.

From condition i) of Definition 4.1 it follows immediately that a payoff vector in the balanced-core lies also in the core of the graph game. Condition ii) states that an element of the core is an element of the balanced-core only if it lies in the intersection of the payoff sets of a graph balanced collection of graphs, i.e. if it is supported by a graph balanced family of graphs. Since each player in $\mathcal{N}$ has an equal total weight in a graph balanced family of graphs, elements in the balanced-core have an appealing stability property. All players have an equal weight in supporting a balanced-core payoff vector, while a balanced-core payoff vector also lies in the payoff set of any graph in the balanced family.

In order to prove the existence of a payoff vector in the balanced-core of a balanced graph game we first introduce an intersection lemma on the $(n-1)$-dimensional unit simplex $\Delta$ defined by

$$
\Delta=\left\{q \in \mathbb{R}_{+}^{n} \mid \sum_{i=1}^{n} q_{i}=1\right\}
$$

\section{Lemma 4.2}

Let $\Gamma \subset \mathcal{G}^{\mathcal{N}}$ be a collection of graphs and for any $G \in \Gamma$, let $C^{G}$ be a (possibly empty) closed subset of $\Delta$ such that

(i) the collection of sets $C^{G}$ covers $\Delta$,

(ii) for any $q$ in the boundary of $\Delta$ it holds that $S \subset\left\{i \in \mathcal{N} \mid q_{i}>0\right\}$ when $q \in C^{G}$ for some $G \in \Gamma \cap \mathcal{G}(S)$.

Then, for any power measure function $m$ there is a graph balanced family $\left\{F^{1}, \ldots, F^{k}\right\}$ of $k$ graphs in $\Gamma$ for which it holds that

$$
\cap_{j=1}^{k} C^{F^{j}} \neq \emptyset .
$$


Before proving the lemma, observe that for a given collection of graphs $\Gamma \subset \mathcal{G}^{\mathcal{N}}$, condition (i) states that the simplex $\Delta$ is covered by closed subsets $C^{G}, G \in \Gamma$. Condition (ii) is some boundary condition. Although no further conditions on the collection $\Gamma$ are required, and hence many elements of $\mathcal{G}^{\mathcal{N}}$ may be missing in $\Gamma$ (or, stated in terms of a graph game, many graphs in $\mathcal{G}^{\mathcal{N}}$ may have empty payoffs sets or are missing in $\Gamma$ ), boundary condition (ii) implies that for any player $i \in \mathcal{N}$ it holds that the single player graph $G_{S}=(S, A(S))$ with $S=\{i\}$ (and therefore $A(S)=\emptyset$ ) is in $\Gamma$. The lemma states that under conditions (i) and (ii) there exists a graph balanced collection of graphs for which the corresponding subsets of $\Delta$ have a nonempty intersection. This generalizes the well-known intersection theorem of Shapley [21], see also [10], in which only sets $C^{S}$ are defined for coalitions $S \subset \mathcal{N}$.

\section{Proof of Lemma 4.2.}

For any $G \in \Gamma$, let us define the vector $c^{G}=\frac{1}{n} e^{\mathcal{N}}-m(G)$. Let $Y^{n}$ be defined by

$$
Y^{n}=\operatorname{conv}\left(\left\{c^{G} \mid G \in \Gamma\right\}\right),
$$

where $\operatorname{conv}(X)$ denotes the convex hull of a set $X \subset \mathbb{R}^{n}$. Observe that $\sum_{j=1}^{n} c_{j}^{G}=0$ for all $G$ and hence $\sum_{j=1}^{n} y_{j}=0$ for all $y \in Y^{n}$. Next, define the correspondence $F: \Delta \rightarrow Y^{n}$ by

$$
F(q)=\operatorname{conv}\left(\left\{c^{G} \mid q \in C^{G}\right\}\right), q \in \Delta .
$$

Since the collection of subsets is a covering of $\Delta$, for every $q \in \Delta$, the set $F(q)$ is nonempty. It is easily verified that, for every $q, F(q)$ is convex and compact. Moreover, $\cup_{q \in \Delta} F(q)$ is bounded and the mapping $F: \Delta \rightarrow Y^{n}$ is an upper hemi-continuous mapping from the set $\Delta$ to the collection of subsets of the set $Y^{n}$. Further, both sets $\Delta$ and $Y^{n}$ are nonempty, convex, and compact. Next, let $H$ be the mapping from $Y^{n}$ to the collection of subsets of $\Delta$ defined by

$$
H(y)=\left\{\widehat{q} \in \Delta \mid q^{\top} y \leq \widehat{q}^{\top} y \text { for every } q \in \Delta\right\}, y \in Y^{n} .
$$

Clearly, for every $y \in Y^{n}$ the set $H(y)$ is nonempty, convex, and compact, and $H$ is upper hemi-continuous. Hence, the mapping $D$ from the nonempty, convex, compact set $\Delta \times Y^{n}$ into the collection of subsets of $\Delta \times Y^{n}$ defined by $D(q, y)=H(y) \times F(q)$ is upper hemi-continuous and for every $(q, y) \in \Delta \times Y^{n}$, the set $D(q, y)$ is nonempty, convex, and compact. According to Kakutani's fixed point theorem the mapping $D$ has a fixed point on $\Delta \times Y^{n}$, i.e. there exist $q^{*} \in \Delta$ and $y^{*} \in Y^{n}$ satisfying $y^{*} \in F\left(q^{*}\right)$ and $q^{*} \in H\left(y^{*}\right)$.

Let $\alpha^{*}=q^{* \top} y^{*}$. From $q^{*} \in H\left(y^{*}\right)$ it follows that $q^{\top} y^{*} \leq \alpha^{*}$ for every $q \in \Delta$. By taking $q=e(i)$, where $e(i)$ is the $i$-th unit vector, we obtain that $y_{i}^{*} \leq \alpha^{*}, i=1, \ldots, n$. Hence,

$$
\left.\begin{array}{ll}
y_{i}^{*}=\alpha^{*} & \text { if } q_{i}^{*}>0, \\
y_{i}^{*} \leq \alpha^{*} & \text { if } q_{i}^{*}=0 .
\end{array}\right\}
$$


Since $\sum_{i=1}^{n} y_{i}^{*}=0$, we obtain that $\alpha^{*} \geq 0$.

On the other hand, $y^{*} \in F\left(q^{*}\right)$ implies there exist nonnegative numbers $\lambda_{1}^{*}, \ldots, \lambda_{k}^{*}$ satisfying $\sum_{j=1}^{k} \lambda_{j}^{*}=1$ and $y^{*}=\sum_{j=1}^{k} \lambda_{j}^{*} c^{F^{j}}$ for a family $\left\{F^{j}, j=1, \ldots, k\right\}$ of graphs such that $q^{*} \in C^{F^{j}}$. Without loss of generality we may assume that $\lambda_{j}^{*}>0$ for every $j=1, \ldots, k$. Let $S_{j} \subset \mathcal{N}$ be the set of players on which $F_{j}$ is defined, i.e. $F_{j}=\left(S_{j}, A\left(S_{j}\right)\right)$ for some set of arcs $A\left(S_{j}\right)$. By the boundary condition we have that $q_{i}^{*}=0$ implies that $i \notin S^{j}$ for every $j=1, \ldots, k$, and thus $c_{i}^{F^{j}}=\frac{1}{n}$. Hence,

$$
y_{i}^{*}=\sum_{j=1}^{k} \lambda_{j}^{*} c_{i}^{F^{j}}=\frac{1}{n}>0 \text { if } q_{i}^{*}=0 .
$$

Suppose there exist an index $i$ such that $q_{i}^{*}=0$. Then it follows from the equations (6) and (7) that $y_{i}^{*}>0$ for all $i$, which contradicts $\sum_{i=1}^{n} y_{i}^{*}=0$. Consequently we have that $q_{i}^{*}>0$ and thus $y_{i}^{*}=\alpha^{*}, i=1, \ldots, n$. Together with $\sum_{i=1}^{n} y_{i}^{*}=0$ this proves that $y^{*}=\underline{0}$. Consequently, $\left\{F^{1}, \ldots, F^{k}\right\}$ is graph balanced. Since $q^{*} \in \cap_{j=1}^{k} C^{F^{j}}$, this completes the proof.

Q.E.D.

The proof of the nonemptiness of the balanced-core of a balanced graph game follows by applying Lemma 4.2 under the conditions stated in the next theorem.

\section{Theorem 4.3 (Nonemptiness Balanced-core)}

A non-transferable utility graph game $(\Gamma, v)$ with power measure function $m$ has a nonempty balanced-core if

(i) for any $i \in \mathcal{N}$, the graph $G_{\{i\}}=(\{i\}, A(\{i\}))$ with $A(\{i\})=\emptyset$ belongs to $\Gamma$ and for some $\alpha_{i} \in \mathbb{R}$, the set $v\left(G_{\{i\}}\right)$ is given by $v\left(G_{\{i\}}\right)=\left\{x \in \mathbb{R}^{n} \mid x_{i} \leq \alpha_{i}\right\}$,

(ii) for every $S \subset \mathcal{N}$ and for every $G \in \Gamma \cap \mathcal{G}(S)$, the set $\left\{\left(x_{i}\right)_{i \in S} \in \mathbb{R}^{S} \mid x \in\right.$ $v(G)$ and $x_{i} \geq \alpha_{i}$ for all $\left.i \in S\right\}$ is bounded,

(iii) for every $G \in \Gamma$, the set $v(G)$ is closed and comprehensive, i.e. if $x \in v(G)$, then $y \in v(G)$ for all $y \leq x$,

(iv) the game is graph balanced.

\section{Proof.}

Without loss of generality we may assume that $\underline{0} \in v\left(G_{\{i\}}\right)$ for any $i \in \mathcal{N}$. To apply Lemma 4.2, we define a closed covering $\left\{C^{G} \mid G \in \Gamma\right\}$ of $\Delta$ satisfying the conditions of the lemma and show that an intersection point of a graph balanced collection of these sets induces an element in the balanced-core of the game. For given $M>0$ and for any $q \in \Delta$, let the number $\lambda_{q}$ be given by

$$
\lambda_{q}=\max \left\{\lambda \in \mathbb{R} \mid-M q+\lambda e^{\mathcal{N}} \in \cup_{G \in \Gamma} v(G)\right\} .
$$


Since $\underline{0} \in v\left(G_{\{i\}}\right)$ and because of conditions (ii) and (iii), $\lambda_{q}$ exists for every $M>0$ and for any $q \in \Delta$. Moreover, following Shapley [21], by condition (ii) $M>0$ can be chosen so large that for every $i \in \mathcal{N}$ and $q \in \Delta, q_{i}=0$ implies that $i \notin S$ for any $S$ such that $-M q+\lambda_{q} e^{\mathcal{N}} \in v(G)$ for some $G \in \Gamma \cap \mathcal{G}(S)$. Now, for $G \in \Gamma$ we define

$$
C^{G}=\left\{q \in \Delta \mid-M q+\lambda_{q} e^{\mathcal{N}} \in v(G)\right\} .
$$

Since every $v(G)$ is closed and comprehensive, the collection of sets $\left\{C^{G} \mid G \in \Gamma\right\}$ is a collection of closed sets covering the simplex $\Delta$, and satisfies the boundary condition of Lemma 4.2. Hence there is a graph balanced family $\mathcal{F}=\left\{F^{1}, \ldots, F^{k}\right\}$ of elements of $\Gamma$ such that $\cap_{j=1}^{k} C^{F^{j}} \neq \emptyset$. Let $q^{*}$ be a point in this intersection, so $q^{*} \in C^{F^{j}}$ for $j=1, \ldots, k$. Then $x^{*}=-M q^{*}+\lambda_{q^{*}} e^{\mathcal{N}}$ lies in $\cap_{j=1}^{k} v\left(F^{j}\right)$, so that $x^{*}$ is a payoff vector supported by the graph balanced family $\mathcal{F}=\left\{F^{1}, \ldots, F^{k}\right\}$.

Since the game is graph balanced we have that $\cap_{j=1}^{k} v\left(F^{j}\right) \subset v^{\prime}(\mathcal{N})$ and hence $x^{*} \in v^{\prime}(\mathcal{N})$. Now, suppose there exist a coalition $S$, a graph $G \in \Gamma \cap \mathcal{G}(S)$ and a vector $y \in v(G)$ such that $y_{i}>x_{i}^{*}$ for all $i \in S$. Since $v(G)$ is comprehensive and cylindric, there is a $\mu>0$ such that $x^{*}+\mu e^{\mathcal{N}} \in v(G)$. However, then $-M q^{*}+\left(\lambda_{q^{*}}+\mu\right) e^{\mathcal{N}} \in v(G)$, which contradicts that $-M q^{*}+\lambda e^{\mathcal{N}} \notin v(G)$ for any $\lambda>\lambda_{q^{*}}$. Hence $x^{*}$ cannot be improved upon by any coalition $S$. Together with the fact $x^{*}$ is supported by the graph balanced family $\mathcal{F}=\left\{F^{1}, \ldots, F^{k}\right\}$ this proves that $x^{*}$ is in the balanced-core of $(\Gamma, v)$.

Q.E.D.

As a result of the theorem we have the following corollaries.

\section{Corollary 4.4}

For some power measure function $m$, let $(\Gamma, v)$ be a graph balanced non-transferable utility graph game satisfying the conditions of Theorem 4.3. Then the core of the game is not empty.

\section{Corollary 4.5}

For some power measure function $m$, let $(\Gamma, v)$ be a graph balanced non-transferable utility graph game satisfying the conditions of Theorem 4.3. Then the core of the induced coalitional game $\left(\mathcal{N}, v^{\prime}\right)$ is not empty.

To conclude this section we remark that in Kamiya and Talman [13] a simplicial algorithm was proposed to find a core element of a coalitional game. Similarly, we can apply the simplicial algorithm on the unit simplex of Doup and Talman [9] to find approximately an element of the balanced-core and so a core element of a balanced graph game. 


\section{$5 \quad$ Noncooperative solutions and graph games}

We consider an industry with two profit maximizing firms producing a homogeneous good. Each firm $i, i=1,2$, has a convex and differentiable cost function $c^{i}\left(q_{i}\right)$, where $q_{i}$ is the quantity produced by firm $i$, with $c^{i}(0)=0$. The demand $q$ for the good is given by the concave and differentiable inverse demand function $p=P(q)$, saying that the total demand for the good equals the total production $q=q_{1}+q_{2}$ when the price $p$ is equal to $P(q)$. So, the profits of the two firms depend on the quantities produced by both firms and are given by the profit functions $\pi^{i}: \mathbb{R}_{+}^{2} \rightarrow \mathbb{R}$ defined by

$$
\pi^{i}\left(q_{i}, q_{j}\right)=q_{i} P\left(q_{i}+q_{j}\right)-c^{i}\left(q_{i}\right), \quad i, j=1,2, j \neq i .
$$

A well-known solution concept for this industrial model is the noncooperative Nash-Cournot solution in which simultaneously each firm chooses an optimal quantity given the choice of the other firm. For given $i=1,2$, let $q_{j} \geq 0$ be the quantity chosen by firm $j \neq i$. Then the optimal reaction of $i$ is the quantity solving the profit maximization problem

$$
\max _{q_{i} \geq 0} q_{i} P\left(q_{i}+q_{j}\right)-c^{i}\left(q_{i}\right) .
$$

Let $r^{i}\left(q_{j}\right)$ be the solution to this problem, i.e. $q_{i}=r^{i}\left(q_{j}\right)$ is the optimal reaction of firm $i$ to the quantity $q_{j}$ set by firm $j$. Under the conditions stated above on the demand and cost functions the two reaction functions $r^{i}: \mathbb{R}_{+} \rightarrow \mathbb{R}_{+}, i=1,2$, are well-defined. A pair $\left(q_{1}^{N}, q_{2}^{N}\right)$ of quantities is a Nash-Cournot equilibrium if

$$
q_{i}^{N}=r^{i}\left(q_{j}^{N}\right), \text { for } i, j=1,2, j \neq i .
$$

We also consider the Stackelberg leader-follower quantity competition game. In this model the follower, say firm $j$, responds with his optimal quantity $r^{j}\left(q_{i}\right)$ to the quantity $q_{i}$ set by the leader $i \neq j$. So, the leader's profit when choosing $q_{i}$ is given by the function $\pi_{S}^{i}: \mathbb{R}_{+} \rightarrow \mathbb{R}_{+}$defined by

$$
\pi_{S}^{i}\left(q_{i}\right)=q_{i} P\left(q_{i}+r^{j}\left(q_{i}\right)\right)-c^{i}\left(q_{i}\right), \quad i=1,2, j \neq i .
$$

For $i=1,2$, a pair of quantities $\left(q_{1}^{*}, q_{2}^{*}\right)$ is a Stackelberg solution of the noncooperative market game with firm $i$ as leader and $j \neq i$ as follower when

(i) $\quad q_{j}^{*}=r^{j}\left(q_{i}^{*}\right)$, and

(ii) $\quad q_{i}^{*}$ solves $\max _{q_{i} \geq 0} \pi_{S}^{i}\left(q_{i}\right)$.

Finally, under the conditions on the demand and cost functions stated above, the profit maximizing problem

$$
\max _{q_{i}} q_{i} P\left(q_{i}\right)-c^{i}\left(q_{i}\right)
$$


of firm $i$ in case this firm could control the market as a monopolist has a unique solution, say $q_{i}^{m}$. Let $M C^{i}: \mathbb{R}_{+} \rightarrow \mathbb{R}$ given by $M C^{i}\left(q_{i}\right)=\frac{\partial c^{i}\left(q_{i}\right)}{\partial q_{i}}$ be the marginal cost function of firm $i, i=1,2$. We now assume that also the following conditions hold.

(i) For both firms $i=1,2$, the Stackelberg leadership profit function $\pi_{S}^{i}$ is concave in $q_{i}$.

(ii) For both firms $i=1,2$, it holds that $P\left(q_{i}^{m}\right)>M C^{j}(0), j \neq i$.

The first condition guarantees that there exists a unique Nash-Cournot equilibrium, see for instance Tirole [22], page 225-226. The second condition, saying that the market price in case firm $i$ operates as a monopolist is higher than the marginal cost of firm $j \neq i$ at $q_{j}=0$, guarantees that at the unique Nash-Cournot equilibrium both firms are on the market, i.e. $q_{i}^{N}>0$ for $i=1,2$. Observe that in a Nash-Cournot equilibrium each player has a nonnegative profit, because a firm always has the possibility to set its production equal to zero, making profit equal to zero. Furthermore, under the first condition also the profit maximizing problem of the leader in the Stackelberg game has a unique solution. In the following we denote this solution with $q_{i}^{S}$, i.e. the pair of quantities $\left(q_{i}^{S}, r^{j}\left(q_{i}^{S}\right)\right)$ is the unique pair of quantities solving the Stackelberg game with firm $i$ as leader, $i=1,2$.

We want to show that the Nash-Cournot model can be formulated as a cooperative graph game. To do so, take $\mathcal{N}=\{1,2\}$ and let $\Gamma \subset \mathcal{G}^{\mathcal{N}}$ be the collection $\left\{G_{1}, G_{2}, G^{1}, G^{2}\right\}$ given by

(i) for $i=1,2, G_{i}=(\{i\}, A(\{i\}))($ with $A(\{i\})=\emptyset)$,

(ii) for $i=1,2, G^{i}=\left(\{1,2\}, A^{i}\right)$ with $A^{i}=\{(j, i) \mid j \neq i\}$.

So, $\Gamma$ is the collection of graphs containing the two one-player graphs and the two twoplayer graphs in which one of the players is dominated by the other player.

Next, we define the sets $v(G)$ for $G \in \Gamma$. Consider first the graph $G^{i} \in \Gamma$, in which player $i$ is dominated by the other player $j$, i.e. the firms play the Stackelberg game with player $j$ as the leader. Therefore we assign to this graph the set of payoffs, which can be obtained when player $j$ takes an arbitrarily chosen quantity $q_{j}$ and player $i$ takes his optimal reaction $q_{i}=r^{i}\left(q_{j}\right)$, i.e.

$$
v\left(G^{i}\right)=\left\{x \in \mathbb{R}^{2} \mid \exists q_{j} \text { such that } x_{j} \leq \pi^{j}\left(q_{j}, r^{i}\left(q_{j}\right)\right), x_{i} \leq \pi^{i}\left(r^{i}\left(q_{j}\right), q_{j}\right)\right\}, i=1,2 .
$$

To define the sets $v\left(G_{i}\right), i=1,2$, we assume that both players are aware that they can get at least the payoff to be realized as a follower in the Stackelberg game. So, recalling that 
$q_{j}^{S}$ is the optimal quantity of $j$ in the Stackelberg game with $j$ as leader, this yields the payoff sets

$$
v\left(G_{i}\right)=\left\{x \in \mathbb{R}^{2} \mid x_{i} \leq \pi^{i}\left(r^{i}\left(q_{j}^{S}\right), q_{j}^{S}\right)\right\}, i=1,2 .
$$

Clearly, condition (i) of Theorem 4.3 is satisfied. Moreover, since $\pi_{S}^{j}$ is decreasing for $q_{j}>q_{j}^{S}$, all sets $v(G), G \in \Gamma$, satisfy conditions (ii) and (iii) of Theorem 4.3. Assigning the positional power measure to the graphs yields the power vectors $m^{p}\left(G_{i}\right)=e(i)$ and $m^{p}\left(G^{i}\right)=e(j), j \neq i$. From this it follows that when $\mathcal{F}$ is a graph balanced family then it holds that $\mathcal{F}$ equals either $\left\{G_{1}, G_{2}\right\}$, or $\left\{G^{1}, G^{2}\right\}$, or $\left\{G_{i}, G^{i}\right\}$ for some $i \in\{1,2\}$. Notice that

$$
\begin{array}{ll}
v\left(G_{1}\right) \cap v\left(G_{2}\right)=\left\{x \in \mathbb{R}^{2} \mid x_{i} \leq \pi^{i}\left(r^{i}\left(q_{j}^{S}\right), q_{j}^{S}\right), i=1,2\right\} & \subset v\left(G^{1}\right) \cup v\left(G^{2}\right), \\
v\left(G^{1}\right) \cap v\left(G^{2}\right) & \subset v\left(G^{1}\right) \cup v\left(G^{2}\right), \\
v\left(G_{i}\right) \cap v\left(G^{i}\right)=\left\{x \in \mathbb{R}^{2} \mid x_{i} \leq \pi^{i}\left(r^{i}\left(q_{j}^{S}\right), q_{j}^{S}\right)\right\} \cap v\left(G^{i}\right) & \subset v\left(G^{1}\right) \cup v\left(G^{2}\right) .
\end{array}
$$

Since $v\left(G^{1}\right) \cup v\left(G^{2}\right)=v^{\prime}(\{1,2\})$, it follows that for any graph balanced family the intersection of the payoff sets is in $v^{\prime}(\mathcal{N})$. Hence the graph game is graph balanced and thus satisfies condition (iv) of Theorem 4.3. So, all conditions of the theorem are satisfied and hence the graph game has a nonempty balanced-core.

Let $x^{*}=\left(x_{1}^{*}, x_{2}^{*}\right)^{\top}$ be a payoff vector in the balanced-core. Then there are three possibilities. First, suppose $x^{*}$ is supported by the graph balanced family $\left\{G_{1}, G_{2}\right\}$, i.e. the collection of the two single player graphs. Then, $x^{*} \in v\left(G_{i}\right)$ and thus $x_{i}^{*} \leq \pi^{i}\left(r^{i}\left(q_{j}^{S}\right), q_{j}^{S}\right)$, $i=1,2$, i.e. each firm gets a payoff at most equal to his profit he gets as follower in the Stackelberg game. This contradicts that $x^{*}$ is in the core of the graph balanced game, since $\pi^{i}\left(r^{i}\left(q_{j}^{S}\right), q_{j}^{S}\right)<\pi^{i}\left(q_{i}^{N}, q_{j}^{N}\right)$ for $i=1,2$ and the vector $\left(\pi^{1}\left(q_{1}^{N}, q_{2}^{N}\right), \pi^{2}\left(q_{2}^{N}, q_{1}^{N}\right)\right)^{\top}$ lies in both $v\left(G^{1}\right)$ and $v\left(G^{2}\right)$. Secondly, suppose $x^{*}$ is supported by $\left\{G_{i}, G^{i}\right\}$ for some $i=1,2$. Then $x^{*} \in v\left(G^{i}\right) \cap v\left(G_{i}\right)$. Because $x^{*} \in v\left(G^{i}\right)$ there exists a quantity $q_{j}^{*}$ such that

$$
x_{j}^{*} \leq \pi^{j}\left(q_{j}^{*}, r^{i}\left(q_{j}^{*}\right)\right) \text { and } x_{i}^{*} \leq \pi^{i}\left(r^{i}\left(q_{j}^{*}\right), q_{j}^{*}\right) .
$$

Because $x^{*} \in v\left(G_{i}\right)$ it follows that

$$
x_{i}^{*} \leq \pi^{i}\left(r^{i}\left(q_{j}^{S}\right), q_{j}^{S}\right)
$$

When $x^{*}$ is in the core (and thus improvement is not possible) we must have that all inequalities hold with equality. Hence

$$
\pi^{i}\left(r^{i}\left(q_{j}^{*}\right), q_{j}^{*}\right)=\pi^{i}\left(r^{i}\left(q_{j}^{S}\right), q_{j}^{S}\right),
$$

implying that $q_{j}^{*}=q_{j}^{S}$ and the payoffs are the Stackelberg leader-follower game payoffs with player $j$ as leader and player $i$ as follower. However, then there exists a payoff vector 
$x$ in the set $v\left(G^{j}\right)$ with $j \neq i$ that dominates $x^{*}$. To see this, observe that the profits of both players increase if player $i$ continues to play $r^{i}\left(q_{j}^{S}\right)$ but player $j$ decreases his quantity from $q_{j}^{S}$ to his optimal reaction $r^{j}\left(r^{i}\left(q_{j}^{S}\right)\right)$ to the reaction $r^{i}\left(q_{j}^{S}\right)$ of $i$ to $q_{j}^{S}$, yielding the payoff vector $x$ with $x_{i}=\pi^{i}\left(r^{i}\left(q_{j}^{S}\right), r^{j}\left(r^{i}\left(q_{j}^{S}\right)\right)\right)>x_{i}^{*}$ and $x_{j}=\pi^{j}\left(r^{j}\left(r^{i}\left(q_{j}^{S}\right)\right), r^{i}\left(q_{j}^{S}\right)\right)>x_{j}^{*}$ in $v\left(G^{j}\right)$. Hence this contradicts that $x^{*}$ is in the core. So, it follows that any balanced-core element is supported by the graph balanced family $\left(G^{1}, G^{2}\right)$, i.e. the collection of the two two-player graphs. Then $x^{*} \in v\left(G^{i}\right), i=1,2$. Because $x^{*} \in v\left(G^{2}\right)$ we have by definition of the set $v\left(G^{2}\right)$ that there exists a quantity $q_{1}^{*}$ such that

$$
x_{1}^{*} \leq \pi^{1}\left(q_{1}^{*}, r^{2}\left(q_{1}^{*}\right)\right) \text { and } x_{2}^{*} \leq \pi^{2}\left(r^{2}\left(q_{1}^{*}\right), q_{1}^{*}\right) .
$$

Analogously, from $x^{*} \in v\left(G^{1}\right)$ it follows that there exists a quantity $q_{2}^{*}$ such that

$$
x_{1}^{*} \leq \pi^{1}\left(r^{1}\left(q_{2}^{*}\right), q_{2}^{*}\right) \text { and } x_{2}^{*} \leq \pi^{2}\left(q_{2}^{*}, r^{1}\left(q_{2}^{*}\right)\right) .
$$

Since $x^{*}$ is in the core (and thus improvement is not possible) we must have that all inequalities hold with equality and hence it follows that

$$
\pi^{1}\left(q_{1}^{*}, r^{2}\left(q_{1}^{*}\right)\right)=\pi^{1}\left(r^{1}\left(q_{2}^{*}\right), q_{2}^{*}\right) \text { and } \pi^{2}\left(q_{2}^{*}, r^{1}\left(q_{2}^{*}\right)\right)=\pi^{2}\left(r^{2}\left(q_{1}^{*}\right), q_{1}^{*}\right) .
$$

From the unicity of the Nash equilibrium this implies that $q_{i}^{*}=r^{i}\left(q_{j}^{*}\right)$ and hence $q_{i}^{*}=q_{i}^{N}$, $i=1,2$. So, the quantities supporting the balanced-core payoff vector are the NashCournot quantities. This gives the following result.

\section{Result 5.1}

Let $x^{*}$ be an element in the balanced-core of the game $(\Gamma, v)$. Then the corresponding quantities $q_{1}^{*}$ and $q_{2}^{*}$ form the unique Nash-Cournot equilibrium for the noncooperative quantity competition game.

The result shows that the Nash-Cournot outcome of the noncooperative game is supported by the balanced-core outcome of an appropriately defined cooperative graph game. In fact, it should be recognized that the noncooperative behavior is modelled in the way the set $\Gamma$ and the the payoff sets corresponding to the graphs in $\Gamma$ are defined.

We also want to consider the Stackelberg leader-follower quantity competition game. To formulate a cooperative graph game yielding the Stackelberg solution with firm $j$ as leader as a balanced-core solution, we take the collection $\Gamma^{j} \subset \mathcal{G}^{\mathcal{N}}$ as the collection of graphs given by

$$
\Gamma^{j}=\left\{G_{1}, G_{2}, G^{i} \text { for } i \neq j\right\},
$$

with $G_{1}, G_{2}$ and $G^{i}$ as defined above and thus $G^{i}$ the two-player graph in which player $i$ is dominated by player $j$. Again, the sets $v\left(G_{1}\right), v\left(G_{2}\right)$ and $v\left(G^{i}\right)$ are defined as in the case 
above and the game $\left(\Gamma^{j}, v\right)$ satisfies conditions (i), (ii) and (iii) of Theorem 4.3. Assigning the positional power measure to the graphs only the families $\left\{G^{i}, G_{i}\right\}$ and $\left\{G_{1}, G_{2}\right\}$ are graph balanced and it follows as above that for both graph balanced families the intersection of the payoff sets is in $v^{\prime}\left(\{1,2\}=v\left(G^{i}\right)\right.$. Hence the graph game is graph balanced and thus also satisfies condition (iv) of Theorem 4.3. Therefore the graph game has a nonempty balanced-core. Again it follows that a payoff vector $x^{*}=\left(x_{1}^{*}, x_{2}^{*}\right)^{\top}$ in the balanced-core can not be supported by the graph balanced family $\left\{G_{1}, G_{2}\right\}$. Hence we must have that $x^{*} \in v\left(G^{i}\right) \cap v\left(G_{i}\right)$. As shown above for the Nash-Cournot case this implies that the payoffs are supported by the quantities $q_{j}^{*}=q_{j}^{S}$ and $q_{i}^{*}=r^{i}\left(q_{j}^{S}\right)$ and hence the payoffs are the Stackelberg leader-follower game payoffs with player $j$ as leader and player $i$ as follower. Observe that in contrast with the Nash-Cournot case these payoffs cannot be improved by a payoff vector in $v\left(G^{j}\right)$ by decreasing the quantity of the leader $j$ because now $G^{j}$ is not in the collection $\Gamma^{j}$. This gives the following result.

\section{Result 5.2}

Let $x^{*}$ be an element in the balanced-core of the game $\left(\Gamma^{j}, v\right)$. Then the corresponding quantities $q_{1}^{*}, q_{2}^{*}$ form the Stackelberg equilibrium for the noncooperative quantity competition game with player $j$ as leader.

\section{Concluding remarks}

In this paper we introduced graph games and proved that the balanced-core of such a game is nonempty if the game is graph balanced. This concept of balancedness is a generalization of the well-known concept of balancedness of coalitions. Analogously the existence result concerning the nonemptiness of the core is more general than for games in coalitional structure. A game in coalitional structure is a special case in the family of games in graph structure. Indeed, when $\Gamma$ is the collection of coalitional graphs, then the graph game is a game in coalitional structure and graph balancedness coincides with coalitional balancedness. In general the induced coalitional game $\left(\mathcal{N}, v^{\prime}\right)$ of a balanced graph game, need not to be coalitionally balanced. Since a graph game and its induced coalitional game have the same core, it follows that graph balancedness of the underlying graph game is a sufficient condition for the nonemptiness of the core. Some applications show a close relationship between cooperative and noncooperative modelling. Therefore the results of this paper contribute to the Nash program. 


\section{References}

[1] R.J. Aumann, The core of a cooperative game without side payments, Transactions of the American Mathematical Society 98 (1961) 539-552.

[2] R.J. Aumann and B. Peleg, Von Neumann-Morgenstern solutions to cooperative games without side payments, Bulletin of the American Mathematical Society 66 (1960) 173179.

[3] M. Behzad, G. Chartrand and L. Lesniak-Foster, Graphs and Digraphs, (Wadsworth, Belmont, California, 1979).

[4] L.J. Billera, Some theorems on the core of an $n$-person game without side payments, SIAM Journal on Applied Mathematics 18 (1970) 567-579.

[5] D. Bouyssou, Ranking methods based on valued preference relations: a characterization on the net flow method, European Journal of Operational Research 60 (1992) 61-67.

[6] R. van den Brink, Relational power in hierarchical organizations, Ph-D Dissertation, Tilburg University, The Netherlands, 1994.

[7] R. van den Brink and R.P. Gilles, A social power index for hierarchically structured populations of economic agents, in: R.P. Gilles and P.H.M. Ruys (eds.), Imperfections and Behavior in Economic Organizations, (Kluwer Academic Publishers, Dordrecht, 1994), p. 279-318.

[8] R. van den Brink and R.P. Gilles, Measuring domination in directed graphs, mimeo, Tilburg University, The Netherlands, 1999.

[9] T.M. Doup and A.J.J. Talman, A new variable dimension simplicial algorithm to find equilibria on the product space of unit simplices, Mathematical Programming 37 (1987) 319-355.

[10] P.J.J. Herings, An extremely simple proof of the K-K-M-S theorem, Economic Theory 10 (1997) 361-367.

[11] T. Ichiishi, Alternative version of Shapley's theorem on closed coverings of a simplex, Proceedings of the American Mathematical Society 104 (1988) 759-763.

[12] T. Ichiishi and A. Idzik, Closed covers of compact, convex polyhedra, International Journal of Game Theory 20 (1991) 161-169. 
[13] K. Kamiya and A.J.J. Talman, Variable dimension simplicial algorithm for balanced games, Journal of Operations Research Society of Japan 34 (1991) 222-228.

[14] G. van der Laan, A.J.J. Talman, and Z. Yang, Cooperative games in permutational structure, Economic Theory 11 (1998) 427-442.

[15] G. van der Laan, A.J.J. Talman and Z. Yang, Intersection theorems on polytopes, Mathematical Programming 84 (1999) 25-38.

[16] R.B. Myerson, Graphs and cooperation in games, Mathematics of Operations Research 2 (1977) 225-229.

[17] J. Nash, Two-person cooperative games, Econometrica 21 (1953) 128-140.

[18] A.S. Nowak and T. Radzik, The Shapley value for n-person games in generalized characteristic function form, Games and Economic Behaviour 6 (1994) 150-161.

[19] A. Rubinstein, Ranking the participants in a tournament, SIAM Journal of Applied Mathematics 38 (1980) 108-111.

[20] P.H.M. Ruys and J.R. van den Brink, Positional abilities and rents on equilibrium wages, in: P.J.J. Herings, G. van der Laan and A.J.J. Talman (eds.), The Theory of Markets, KNAW Proceedings, (North-Holland, Amsterdam, 1999), p. 261-279.

[21] L.S. Shapley, On balanced games without side payments, in: Mathematical Programming, eds. T.C. Hu and S.M. Robinson (Academic Press, New York, 1973) pp. 261-290.

[22] J. Tirole, The Theory of Industrial Organization, MIT Press, 1988. 